\title{
A vascularized bone-on-a-chip model development via exploring mechanical stimulation for evaluation of fracture healing therapeutics
}

\author{
Bodhisatwa Das ${ }^{1,2}$ (C) Sundeep V. Seesala ${ }^{2} \cdot$ Pallabi Pal $^{2,3} \cdot$ Trina Roy $^{2} \cdot$ Preetam Guha Roy $^{2,4} \cdot$ Santanu Dhara ${ }^{2}$
}

Received: 28 June 2021 / Revised: 25 September 2021 / Accepted: 28 September 2021 / Published online: 29 October 2021

(c) The Author(s), under exclusive licence to Springer Nature Switzerland AG 2021

\begin{abstract}
Bone is the major connective tissue maintaining the structural integrity of the human body. However, fracture and many skeletal degenerative diseases can compromise this function. Thus, therapeutics related to bone degeneration are of significant research interest and require good in vitro models for such therapeutic evaluation. Bone is a highly vascularized tissue and incorporation of this feature is significantly important for mimicking the osteogenic microenvironment. In the current study, we developed a vascularized flat bone model via simultaneous mechanical actuation of mechanical strain and fluid shear. The mechanical strain was achieved by static magnetic field actuation of a magnetic nanocomposite scaffold. The fluid shear was generated by developing a micropattern on the magnetic nanocomposite via replica molding and laser-based microfabrication. From the live cell imaging window of the microdevice, both bone and vasculature like cellular morphology was observed. The SEM study showed thick ECM deposition in the dynamic culture. In the PCR study, both osteogenic (Col1 , osteocalcin) and angiogenic phenotypes (PECAM) were observed in the dynamic culture scaffolds while chondrogenic marker (Col-2) was downregulated.
\end{abstract}

Keywords Bone $\cdot$ Fracture healing $\cdot$ Mechanical strain

\section{Introduction}

Bone is the major load-bearing tissue of the human body that has a combination of collagen and calcium-rich extracellular matrix (ECM) and bone cells which also promotes locomotion and joint movement and controls mineral homeostasis. [1] It is also a niche for bone marrow-derived stem cells and major progenitors of blood cells.[2] Bone is majorly

Bodhisatwa Das

bodhisatwa.das.1985@gmail.com;

bodhisatwa.das@iitrpr.ac.in

Santanu Dhara

sdhara@smst.iitkgp.ernet.in

1 Department of Biomedical Engineering, Indian Institute of Technology Ropar, Rupnagar, India

2 School of Medical Science and Technology, Indian Institute of Technology Kharagpur, Kharagpur, India

3 Department of Chemical Engineering, Purdue University, West Lafayette, IN, USA

4 Department of Biosystems Science and Engineering, Eidgenössische Technische Hochschule Zürich, Zürich, Switzerland composed of osteoblasts (bone-making cells), osteoclasts (bone-degrading cells), thick calcified ECM, and very rich supply of vasculatures. Bone diseases like osteoporosis, Paget's disease, osteomyelitis, rickets, and many other diseases affect this tissue and often lead a patient's structural and functional deformities. Understanding the effect of drugs on bone diseases is significantly important and often it is difficult to explore animal models.

With significant improvements in microfabrication techniques and biomaterial sciences in the last decade, effective development of in vitro models mimicking different organs has been observed. [3] Top-down approaches such as microfluidics and bottom-up approaches such as 3D bioprinting have developed in vitro models significantly capable of mimicking in vivo systems. There are ample studies available in the literature, which have been objected towards simulating the properties of native bone and its diseases. [4] Coculture-based in vitro bone models already have been explored to show the interplay between osteoblast and osteoclasts for understanding bone remodeling. [5, 6]Bone is an organ with one of the richest blood supply in the body. Hence, a major need for mimicking bone microenvironment in vitro is development of blood vessels. The incorporation 
of microfluidics into the system can mitigate this limitation of currently available bone models. Coculture of mesenchymal stromal cells (MSCs) and endothelial cells into microfluidic platforms helped in forming vascularized bone models in vitro. [7, 8] Bone diseases such as osteosarcoma, cancerous metastasis in bone, and osteomyelitis (bone infection) models have been significantly established for drug screening in vitro platforms. [9-14].

Organ-on-a-chip models require to provide different biophysical stimuli (electric field, mechanical stimulation etc.). While the entire dynamic system mostly works on the functionality of mechanical pumping systems, application of biophysical stimuli is mostly performed via incorporating direct pneumatic system or chemically deposited electrodes. However, these systems are difficult to operate as the device works with cell culture media which is highly conductive. Non-invasive actuation like piezo electric and magnetic actuation is observed to be of recent research interest for microsystem researchers. $[15,16]$ Magnetic actuators are easy to operate and can be used to generate strain in a controlled manner for mechanical stimulation. Recently, magnetic actuation-based mechanotransduction has been explored in organoid culture, glial cell culture, and canceron-a-chip models. [17, 18] We also recently reported iron oxide nanoparticle biopolymer 3D-printed nanocomposites can show osteogenic differentiation of MSCs upon external magnetic actuation in vivo. In our device, also we have explored the possibility of magnetic actuation for osteoblast like cellular phenotype establishment.

While a lot of studies focusing on in vitro models of bone cancer, bone marrow, and long bone have been carried out, flat and irregular bones were relatively neglected for in vitro model establishments. [19-22] However, trabecular flat bones take significant roles in the mechanostability of the skeleton. While they are irregular in shape and mechanically anisotropic, they take away the load from the joints and distribute them among the entire skeletal structure. [23] Diseases like osteomyelitis and Paget's disease can affect their mechanical properties and mineralization leading to mechanical failure and skeletal deformities. [24].

Nature has its method of cellular differentiation and multicellular tissue formation via biophysical and biochemical actuation. It has been observed that fluid shear stress upon MSCs can lead them towards endothelial lineage. [25] Mechanical loading and strain have been observed to be effective for the differentiation of MSCs into the osteogenic lineage. [26, 27] In the current study, we have constructed a polyacrylic-based laser-engraved microfluidic device. A micropatterned magnetic scaffold has been explored for simultaneous fluid shear and magnetic actuation-induced mechanical strain to obtain both osteogenic and endothelial phenotypes in the same platform. The shear stress is generated via gradient development in media flow and mechanical strain is achieved by external magnetic actuation on a magnetic nanocomposite (ultrasmall super paramagnetic iron oxide nanoparticle/USPION and gelatin blend).

\section{Materials and methods}

All the materials are used as procured and not purified until mentioned. De-ionized (DI) water was used for all the work. $\mathrm{NaOH}$, sodium citrate $\left(\mathrm{Na}_{3} \mathrm{C}_{6} \mathrm{H}_{5} \mathrm{O}_{7}\right), \mathrm{HCl}, \mathrm{FeCl}_{3}, \mathrm{FeCl}_{2}$, gelatin (porcine skin), glutaraldehyde, and poly-1-lysine were purchased from Merck, India. Commercially available polyacrylic sheets of thickness $1.5 \mathrm{~mm}$ and $3 \mathrm{~mm}$ and $0.2 \mathrm{~mm}$ were used. 2.5 -mm-inner-diameter silicone rubber tubes and 1-mm-thick silicone rubber sheets were procured from the market. 316 stainless steel hex bolts (approximately $8 \mathrm{~cm}$ in length and $2 \mathrm{~cm}$ in diameter) and matching nuts were also purchased commercially.

\section{Synthesis and characterization of USPIONs}

The citrate-capped USPION (ultrasmall super paramagnetic iron oxide nanoparticles) synthesis protocol was inspired by the method described elsewhere with certain modifications. [28] $\mathrm{FeCl}_{2}$ powder was added to $10 \mathrm{mM}$ sodium citrate $\left(\mathrm{Na}_{3} \mathrm{C}_{6} \mathrm{H}_{5} \mathrm{O}_{7}\right)$ solution ( $\mathrm{pH}$ raised by $\mathrm{NaOH}$ solution to 11 and heated to the temperature of $70{ }^{\circ} \mathrm{C}$ ) so that the final concentration of $\mathrm{FeCl}_{2}$ remains $1 \mathrm{mM}$. In that warm alkaline solution, equal volume $2 \mathrm{mM} \mathrm{FeCl}_{3}$ solution was added dropwise. The solution turns brownish-black. The solution was cooled to room temperature and neutralized by $1 \mathrm{M} \mathrm{HCl}$. The nanoparticles were collected by centrifugation (1000 RCF) and dried in a vacuum oven. The synthesized USPIONs were characterized with HRTEM (JEOL, JEM-2100), XRD Bruker (Cu-K $\alpha$ ), and SQUID (Quantum Design, USA).

\section{Fabrication of USPION/gelatin nanocomposite micropatterned scaffold}

The scaffold fabrication slurry was prepared by mixing porcine skin gelatin $(20 \% \mathrm{~W} / \mathrm{V})$ in warm DI water. It was mixed with a mechanical stirrer continuously to prepare a homogenous slurry having a USPION concentration $(10 \mathrm{mg} /$ $\mathrm{mL}$ ). The slurry was allowed to mix overnight, and bubbles were diminished by adding n-octanol before casting. The polyacrylic molds (3-mm plates) were prepared by a tabletop computer-controlled laser machine (ULS 2.30, Universal Laser System, UK). The mold had semicircular channels engraved to have features of $200 \mu \mathrm{m}$ (final diameter) and approximate surface roughness is $20-30 \mu \mathrm{m}$; both are important for creating fluid shear gradient (the entry and exit channel in the bioreactor are approximately $1 \mathrm{~mm}$ in diameter 
and a scaffold is mostly comprising of 6 microchannels). The slurry was poured on the mold $(50-\mathrm{mL}$ slurry for a composite comprising of 20 scaffolds having a dimension of length $1 \mathrm{~cm}$, width $1 \mathrm{~cm}$, and thickness $0.5 \mathrm{~cm}$ ) slowly and allowed to solidify in vacuum at $4{ }^{\circ} \mathrm{C}$ for $2 \mathrm{~h}$. After that, the composites were peeled out of the mold carefully. The composite scaffolds were cross-linked using cold 5\% glutaraldehyde $(\mathrm{V} / \mathrm{V})$ in $\mathrm{DI}_{2} \mathrm{O}$ in the dark environment for $6 \mathrm{~h}$. The scaffolds were further treated with $10 \%$ glycine solution for neutralizing the unreacted aldehyde groups which could be potentially toxic for the biological system. The scaffolds were washed thoroughly with DI $\mathrm{H}_{2} \mathrm{O}$ thrice and dried in a vacuum oven putting some weight on the sample to prevent the samples from buckling while drying (Fig. 1).

The scaffold fabricated was characterized using SEM (Carl Zeiss, EVO 60), Auger electron spectroscopy (ULVAC, PHI), nanoindentation (TI 950 TriboIndenter, Hysitron Inc., USA), and 3D optical surface profilometry (Bruker). The swelling ratio of the samples was measured by finding out the weight of the dry sample, putting the samples in $\mathrm{DI} \mathrm{H}_{2} \mathrm{O}$, and measuring weight over a different period of wet samples. The swelling ratio is measured by the following formula:

SwellingRatio $=\left(\boldsymbol{W}-\boldsymbol{W}_{0}\right) / \boldsymbol{W}_{0} * 100\left[W=\right.$ wet weight $; W_{0}=$ dry weight $]$

\section{Fabrication of microfluidic device}

The microfluidic device was fabricated according to the scheme presented in Fig. 2. Polyacrylic sheets (3-mm thickness) were procured from the market. These were machined using a $\mathrm{CO}_{2}$ laser machine (ULS 2.30, Universal Laser System, UK) into different layers. 2D CAD models were created and exported to fabricate microchannel, scaffold attachment spots, and glass windows for live-cell imaging. The sealing of the device was done by silicone rubber membranes ( $1 \mathrm{~mm}$ thick) and the entire device was firmly attached by 360 SS stainless steel screws. Silicone rubber tubing with gas permeability was utilized for media exchange and micro-infusion pumps were used flowing of the media. Plates were fabricated using laser machining including two cover plates, three structural plates (one top, one middle, and one bottom, refer to Fig. 2). The top and bottom plates have scaffold support areas and media entry and exit points. They are attached to a glass window for microscopy. The middle plate has the microfluidic channel for media transport and scaffold chambers. For sealing, silicone rubber membranes (1-mm thickness) are cut into shapes. The entire device is strongly tightened by stainless steel screws and bolts. The top and bottom chambers are covered with glass coverslips which were attached by polyacrylic dissolved with chloroform. A perfusion pump (Masterflex, Cole Parmer India) is used for media persusion.

The entire setup is kept in the $\mathrm{CO}_{2}$ incubator $\left(37^{\circ} \mathrm{C}, 5 \%\right.$ $\mathrm{CO}_{2}$ ) for the complete study (2-3 weeks) except time-totime checking under the microscope (please find the video submitted with the manuscript).

\section{Cell culture and imaging study}

The cell culture study was conducted by culturing mesenchymal stromal cells (MSCs from Wharton's Jelly, 50,000 cells/sample) on the magnetic composite scaffolds at $37^{\circ} \mathrm{C}$ and $5 \% \mathrm{CO}_{2}$ using DMEM high glucose,
Fig. 1 The fabrication strategies for microchanneled magneto-active USPIONgelatin nanocomposite by laser micromachining, replica molding, and cross-linking to prepare magneto-active 3D scaffold with microchannels

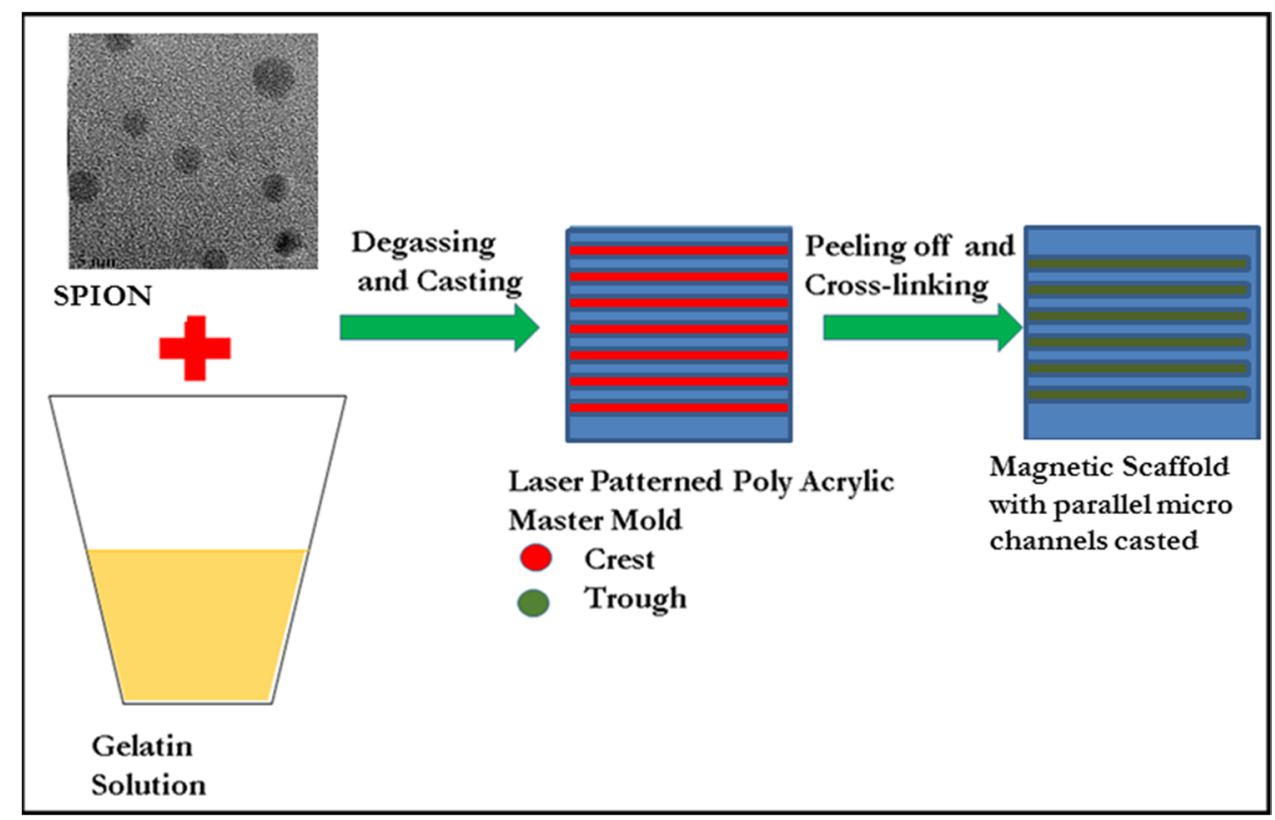


Fig. 2 The in-house fabricated bioreactor with in situ bio-imaging setup (for operation, please find the video file submitted). Polyacrylic sheets were micromachined by a laser engraving machine and they were joined by stainless screws and sealed by silicone rubber sheets cut into shapes. Microscopy windows were prepared by attaching glass coverslips

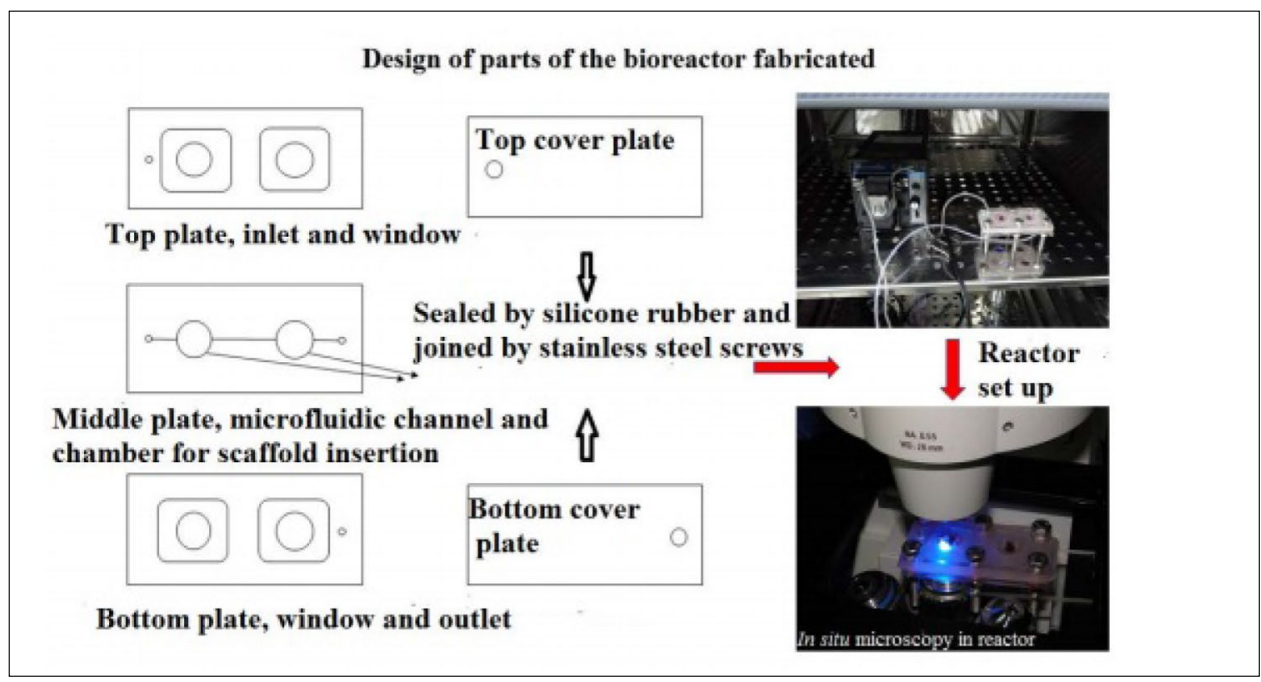

$10 \%$ FBS, and $2.5 \%$ antimicrobial and antifungal solution (HiMedia). A higher concentration of antimicrobial agent application was used to minimize possibilities of infection in the bioreactor setup. $10^{5}$ cells per scaffold were cultured. After $72 \mathrm{~h}$, the scaffolds were transferred into the bioreactor, and media was flown through the samples with a flow rate of $1 \mathrm{~mL} / \mathrm{h}$ using a medical-grade infusion pump. Two 0.05-T magnetic strength rare earth metal bar magnets $\left(1 \mathrm{~cm}^{2}\right.$ area and $2-\mathrm{mm}$ thickness) were attached to both sides of the reactor for magnetic actuation. The magnets were attached to the sides of the reactor chamber so that the center of the magnets remains at an angle of approximately $38^{\circ}$ with the center of the scaffold (Fig. 3). The control experiment for cell culture was conducted by culturing the cells on scaffolds in 12-well cell culture plates (Nunc). The microscopic analysis was conducted in both SEM (Carl Zeiss, EVO 60) and fluorescence microscopy (Carl Zeiss). The long-term cell tracking study was conducted using an in-house prepared cell tracking dye (carbon nano dots, detailed protocol mentioned in supplementary section). [29, 30].

\section{RT-PCR study}

The MSCs were cultured on the scaffolds $\left(10^{5}\right.$ cells/scaffolds) and four different groups were prepared (FMg, fluid shear + magnetic actuation; F, only fluid shear; $\mathrm{Mg}$, only magnetic actuation; and control). After 15 days, the experiments are completed and cells were isolated from scaffolds. Total RNA was isolated from the samples after 15 days of culture using an RNA extraction kit (Genetix, India) following the manufacturer's protocol. RNA concentration was balanced via measuring in "Nanodrop" (Bio-Rad). RevertAid First Strand cDNA Synthesis Kit (Thermo Fisher Scientific) was utilized for cDNA synthesis following the manufacturer's protocol.
PCR amplification was done for 35 cycles using the following conditions: $94{ }^{\circ} \mathrm{C}$ (denaturation) for $30 \mathrm{~s}$, variable 1 (annealing) for $30 \mathrm{~s}$, and $72{ }^{\circ} \mathrm{C}$ extensions for $30 \mathrm{~s}$ and a final extension at $72{ }^{\circ} \mathrm{C}$ for $10 \mathrm{~min}$ in the thermal cycler (Eppendorf, Germany). PCR products were then analyzed by $1 \%$ agarose gel electrophoresis. It was watched via adding ethidium bromide solution into the gel using Geldoc (Bio-Rad) machine. The bands were analyzed using ImageJ software and relative band intensities were plotted. GAPDH was utilized as a housekeeping gene and its expression was checked. The other gene expression, which were considered into account, are collagen-I, collagen-II, BMP-2, PECAM, and osteocalcin (primer sequences and melting temperature are provided in Table 1).

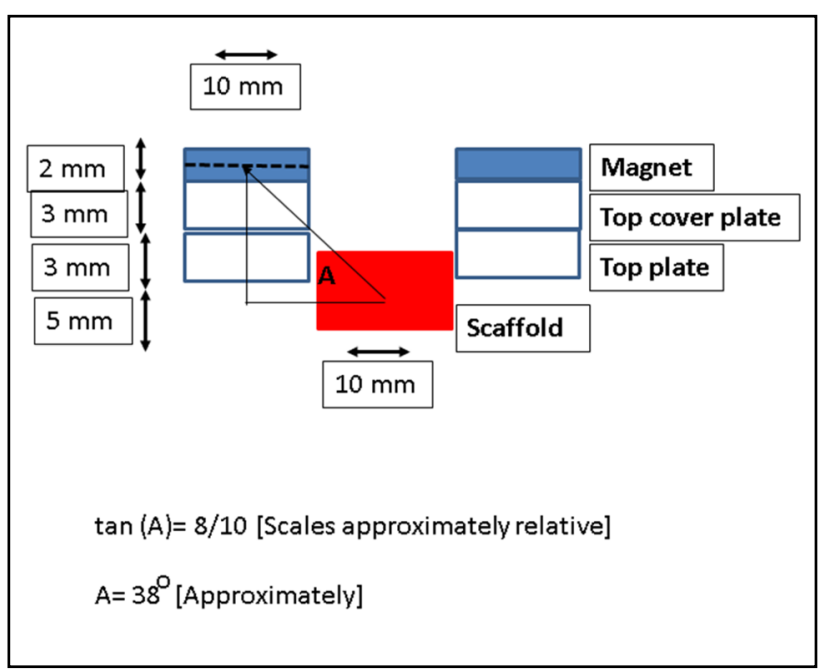

Fig. 3 Static bar magnet-based scaffold actuation system (2 rare earth bar magnets of magnetic strength $0.05 \mathrm{~T}$ was used) 


\section{Statistical analysis}

Statistical analysis was conducted by taking the mean and standard deviation of samples run in triplicates $(n=3)$. The Student's $t$-test is conducted with $p \leq 0.05$ to be statistically significant concerning control wherever possible.

\section{Results}

\section{Physicochemical characterization of the nanoparticles}

The synthesized nanoparticles were characterized using TEM, XRD, and SQUID analysis (Fig. 4). The nanoparticles were observed to have mostly spherical in shape and approximately $5.2 \pm 1.2 \mathrm{~nm}$. The inter crystal plane distance

Table 1 List of primer sequences and respective melting temperature

\begin{tabular}{llll}
\hline Gene name & Forward primer sequence $\left(5^{\prime}-3^{\prime}\right)$ & Reverse primer sequence $\left(5^{\prime}-3^{\prime}\right)$ & $\begin{array}{l}\text { Melting } \\
\text { temperature } \\
\left({ }^{\circ} \mathrm{C}\right)\end{array}$ \\
\hline Collagen-I & GCGCCAGAAGAACTGGTACATCAGCAA & GCGCGCCATACTCGAACTGGAATC & 60 \\
Osteocalcin & ATGAGAGCCCTCACACTCCTC & GCCGTAGAAGCGCCGATAGGC & 58.5 \\
GAPDH & CCATGGAGAAGGCTGGGG & CAAAGTTGTCATGGATGACC & 54 \\
Collagen -II & GGCAATAGCAGGTTCACGTACA & CGATAACAGTCTTGCCCCACTT & 53.9 \\
PECAM/CD-31 & TCAAATGATCCTGCG & CCACCACCTTACTTG & 58.9 \\
BMP-2 & ACCCGCTGTCTTCTAGCGT & CTCAGGACCTCGTCAGAGGG & 57 \\
\hline
\end{tabular}

Fig. 4 Physicochemical characterization of the USPIONs: (a) TEM brightfield image, (b) HRTEM images showing inter crystalline planes of the nanostructures, (c) XRD pattern of the USPIONs to confirm its phases, (d) SQUID analysis of the USPIONs for finding the magnetic properties
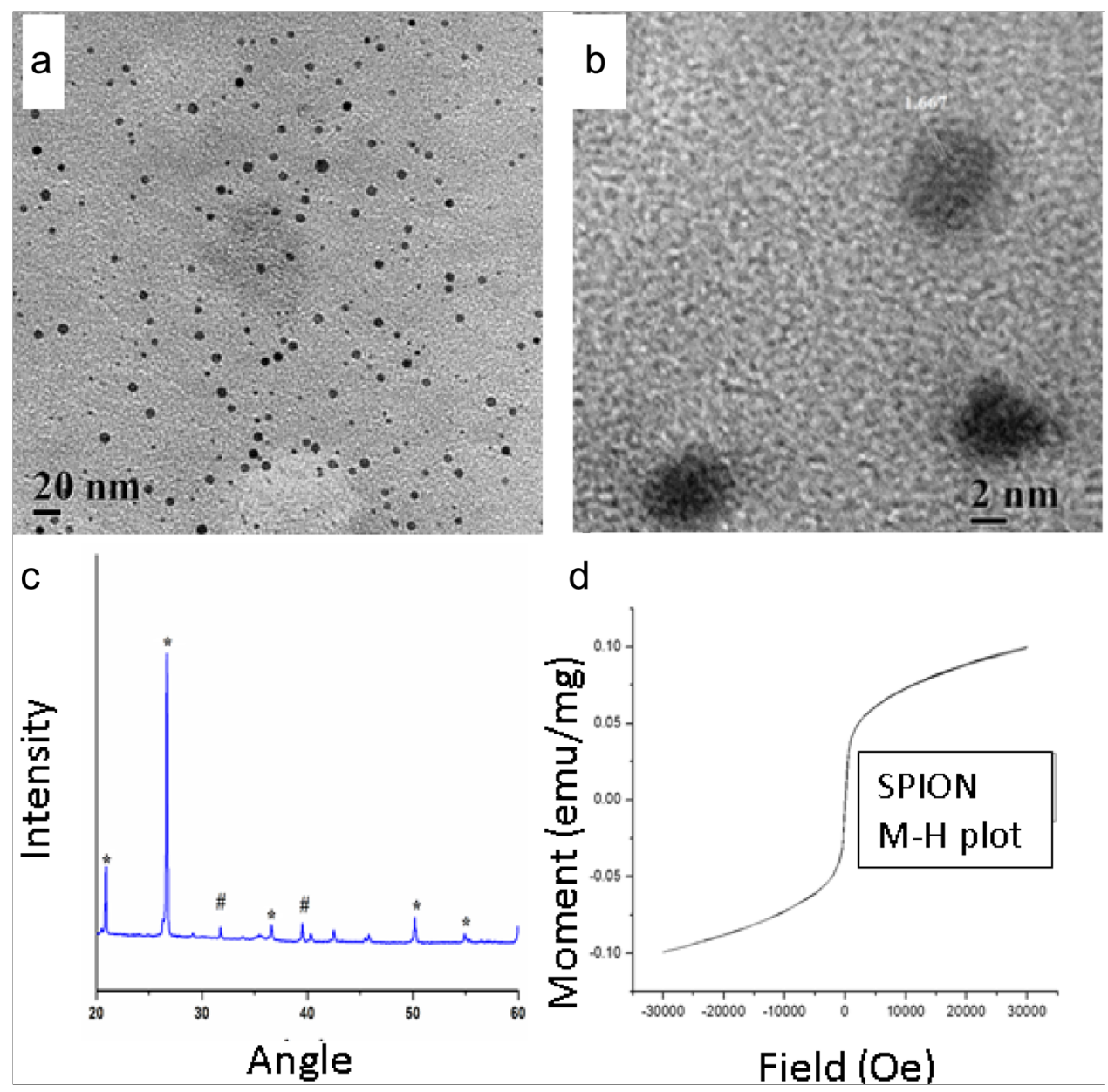
from the high resolution was observed to be $1.67 \dot{\mathrm{A}}$. The XRD analysis suggested presence of mixed phases in the nanoparticles.

The peaks around $26^{\circ}$ and $22^{\circ}$ can be attributed to $\mathrm{Y}-\mathrm{Fe}_{2} \mathrm{O}_{3}$. The peaks around $31^{\circ}, 35^{\circ}$, and $39^{\circ}$ could be attributed to $\mathrm{Fe}_{3} \mathrm{O}_{4}$. The peaks around $41^{\circ}, 45^{\circ}$, and $51^{\circ}$ can be attributed to $\alpha-\mathrm{Fe}_{2} \mathrm{O}_{3}$. [31] The SQUID analysis suggested the material has significant magnetic properties as per our previous report of carbon dot-assisted SPION synthesis. [32] Thus, the magnetic nanocomposite developed using these particles can be used for mechanical actuation using external magnetic field. The gelatin-USPION composite was prepared by pouring the slurry on the micropatterned mold using replica molding. The surface roughness (Fig. 5a and b) in two orthogonal axes $(X$ and $Y$ ) is plotted. The average roughness $(\Delta Z)$ was observed to be in the range of $220 \mathrm{~nm}$ which is helpful in cellular attachment.33 The swelling study was conducted to understand the final dimensions of the microchannels prepared in the scaffolds (Fig. 5c). Considering, there is approximately $55 \%$ of swelling, the final channel diameters at steady state should be in the range of $300-350 \mu \mathrm{m}$. The SEM analysis shows that the scaffolds have microchannels in the range of $220 \pm 30-\mu \mathrm{m}$ diameter (Fig. 5d).

The surface chemical characterization of the samples was conducted by AES which reaches until first few nanometers depth of the sample. While gelatin being a denatured protein (collagen) having a random distribution of carbon and oxygen (Fig. 6a and b), the iron nanoparticles (Fig. 6c) were observed to be homogenously distributed. The samples' micromechanical property was observed to be in the range of human trabecular bone (Figure S3, modulus $9.88 \pm 2.06 \mathrm{GPa}$ and hardness $0.365 \mathrm{GPa}$ ). [34] The live cell microscopy results showed spindle-like structures in the presence of mechanical stress (Fig. 7a) and formation of bone-like nodular structures (Fig. 7b). We also observe thick ECM deposition (Fig. 7c). However, endothelial cell-like tubular structural features also (Fig. 7d-f) have been observed which suggests presence of both phenotypes (bone cells and endothelial cells). This observation was further verified using PCR analysis (Fig. 8). In PCR, 4 different types of samples were denoted ( $\mathrm{F}$ is only fluid shear, $\mathrm{Mg}$ is only magnetic field, FMg is both fluid shear and magnetic field, and $\mathrm{CN}$ is static culture with no magnetic field). It is observed that PECAM (endothelial marker) and BMP-2 were highest in F, whereas OCN (osteocalcin, bone tissue marker) was observed to be highest in Mg. A housekeeping gene (GAPDH) and positive control Coll-2 (chondrocyte marker) were shown to have similar levels of expression suggesting effect of physical forces only modulates bone and endothelial phenotype. Interestingly, the PCR result of FMg in the major ECM protein Col-1 is highest suggesting highest ECM synthesis. This result is as per the SEM result observed in cell culture studies, as just like upregulation of Col-1, very high amounts of ECM deposition are observed (Fig. 7c and supplementary results: Figure S1). But gene expression of OCN, BMP-2, and PECAM in FMg is next to highest. This result suggests the combination of fluid shear $(\mathrm{F})$ and magnetic field $(\mathrm{Mg})$ is capable of creating both osteogenic and endothelial phenotypes in a single model. Similar results were obtained from H\&E histological imaging (supplementary section figure $\mathrm{S} 2$ ).

\section{Discussion}

Organ-on-a-chip is a concept mostly explored to mimic in vivo tissue models in vitro. Bone is the major load-bearing connective tissue of the mammalian body as well as it is working as the niche for many hematopoietic cells. Therefore, a biomimetic in vitro model for osteogenic tissue is of significant research. Specifically, the pharmaceutical industries working in the area of osteogenic diseases such as rickets, osteoporosis, osteoarthritis, and orthopedic trauma are highly interested in the development of in vitro bone models. [35] While there are certain in vitro bone models that exist in literature, most of them fail to mimic multiple features. Bone is a mechanoresponsive tissue. Therefore, the mechanical stimulus must have a specific effect on the system. Secondly, bone is significantly rich is vasculature which helps in nutrient and gaseous transport. Most of the in vitro models available in literature depend upon diffusion for this transport phenomenon. From Fick's law of diffusion, it can be denoted that

$\mathbf{J}=\mathbf{D A}(\mathbf{C} 1-\mathbf{C} 2) / \mathbf{L}$

( $\mathbf{J}$ is the flux density (diffusion rate; $\mathrm{mol} / \mathrm{m}^{2} / \mathrm{s}$ ); $\mathbf{D}$ is the diffusion coefficient (function of molecule and medium); $\mathbf{A}$ is the cross-sectional area; $\mathbf{C 1}$ and $\mathbf{C 2}$ are the concentrations at source and sink; $\mathbf{L}$ is the path length, and $\mathbf{D}$ depends inversely upon density of the medium) [36].

As the flux density depends inversely upon the path length of diffusion, for a thick matrix such as bone, the maximum limit of $\mathbf{L}$ for effective diffusion is $150-200 \mu \mathrm{m}$.

Therefore, lack of vasculature beyond this distance can be considered as a major limitation to the mass transport phenomena of an in vitro bone model. From the data provided in the figures, it is clear that the channels are around $250 \mu \mathrm{m}$ in dimensions with the distance between channels to be around $50 \mu \mathrm{m}$ (Fig. 5d). From $1 \mathrm{~mm}$ at the entry to the division of the flow to $200 \mu \mathrm{m}$ generates a shear gradient.

Now, from Newton's law of viscosity, we get

$\tau=-\mu \Delta / \Delta z$

( $\tau$ is the shear stress, $\boldsymbol{\mu}$ is the coefficient of viscosity $(0.78$ $* 10^{-3} \mathrm{~N} . \mathrm{s} / \mathrm{m}^{2}$ ) for DMEM, $\mathrm{u}$ is the velocity, and $\mathbf{z}$ is the distance traversed)

$\Delta u=-0.2231 \mathrm{~m} / \mathrm{s}, / \Delta z=0.005 \mathrm{~m}$ 


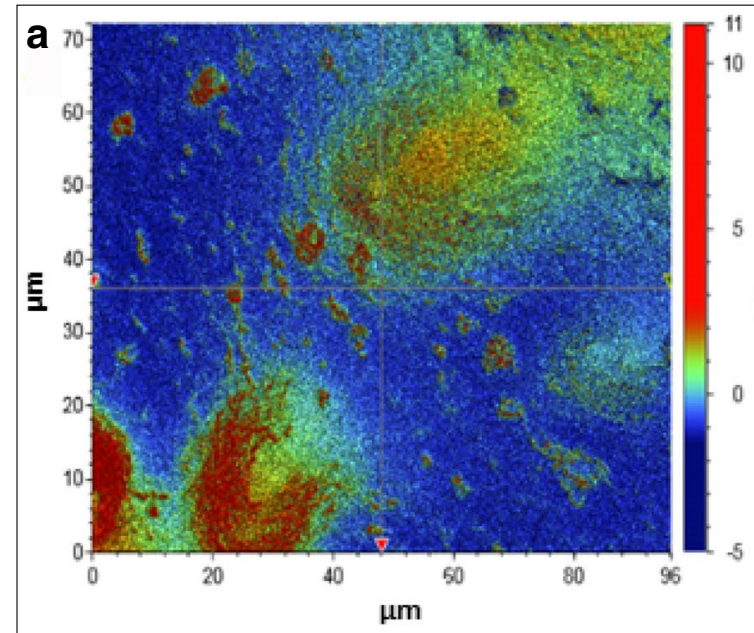

b
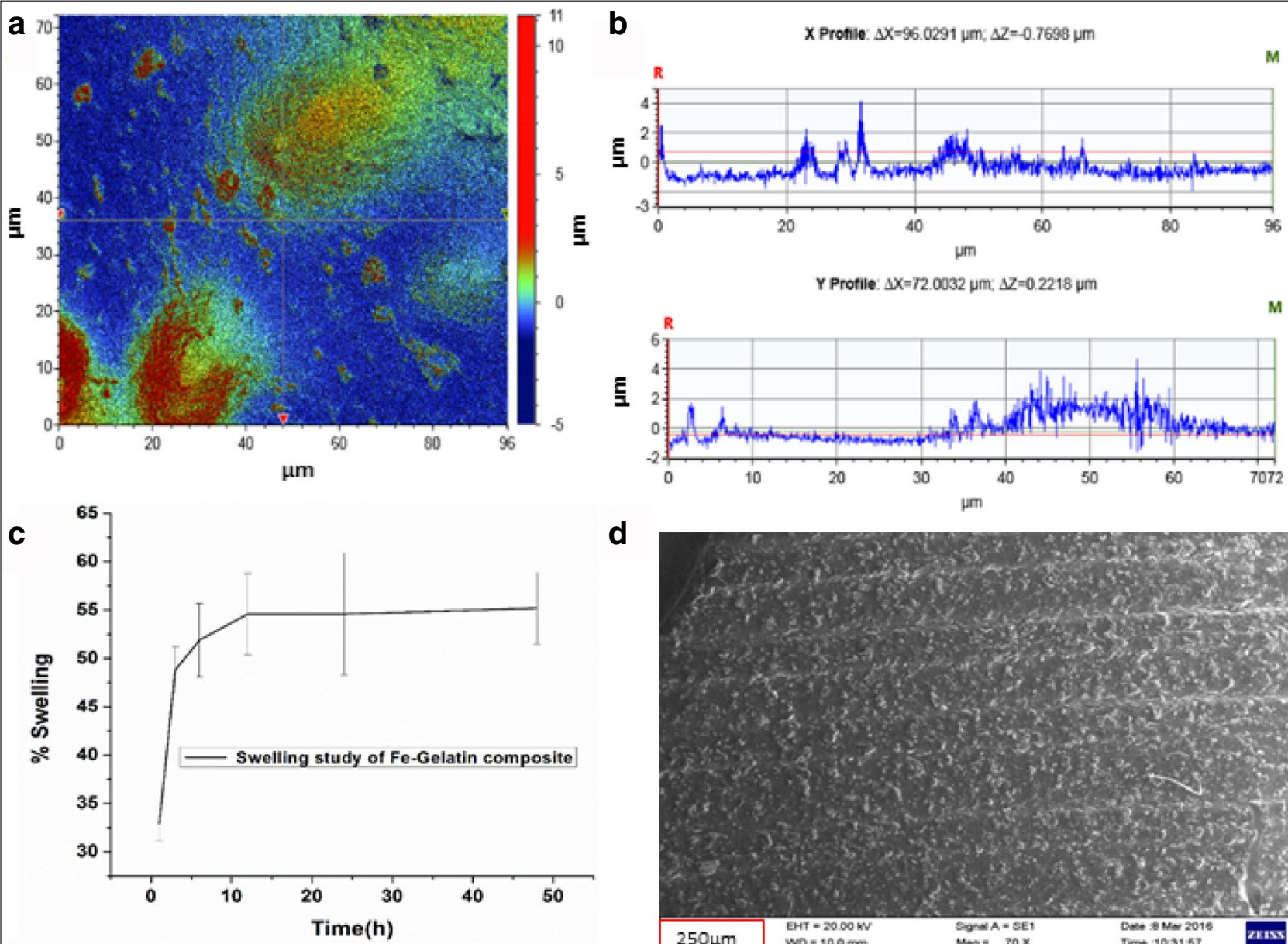

d

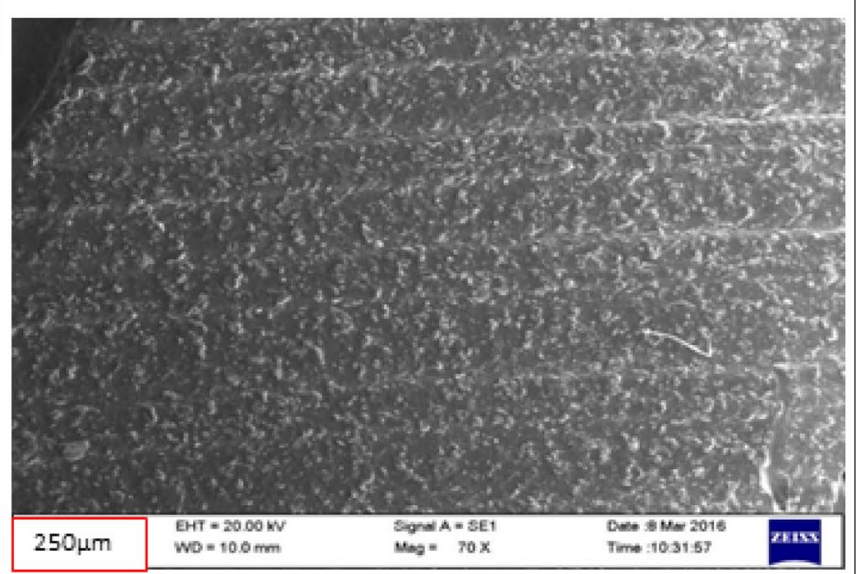

e

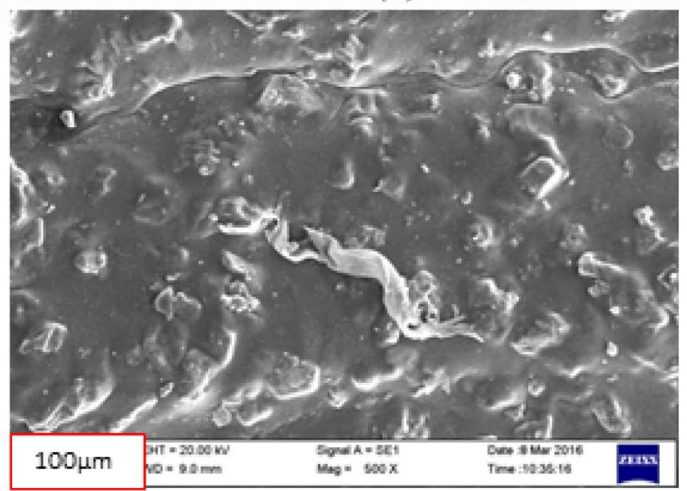

(d) SEM image to show casted microchannels (scale $250 \mu \mathrm{m}$ ). (e)

Fig. 5 Physicochemical characterization of the scaffolds with microfluidic channels. (a) 3D surface profilometry images showing microand nanoroughness. (b) 3D surface roughness profile along the $X$ and $Y$ axes. (c) Swelling study until the scaffold reaches to equilibrium.

Magnified SEM image to show the microroughness on the surface (scale $100 \mu \mathrm{m}$ )

$\tau=0.35 \mathrm{~N} / \mathrm{m}^{2}$ which is at the similar level as observed with previous literature where people observed the effect of shear stress upon endothelial differentiation to enhance endothelial-like differentiation up to 11 to 214 fold. [37] At the same time, magnetic field working upon the scaffold (0.05-T two bar magnets) allows the scaffold to experience mechanical stretch. The mechanical stretch was generated by magnetic actuation where the magneto-active scaffold (the scaffold's SQUID result is provided suggesting the magnetic susceptibility of the biopolymer-magnetic nanoparticle composite) was kept between two equally strong rare earth metal permanent bar magnets $(0.05 \mathrm{~T})$. The scaffold was stuck to its position in the reactor with sterile 
Fig. 6 Surface chemical characterization of the scaffold using AES: (a) $\mathrm{C} 1,(\mathbf{b}) \mathrm{O} 1,(\mathbf{c})$ $\mathrm{Fe} 1$, (d) combined showing the distribution of different atoms on the scaffold surface
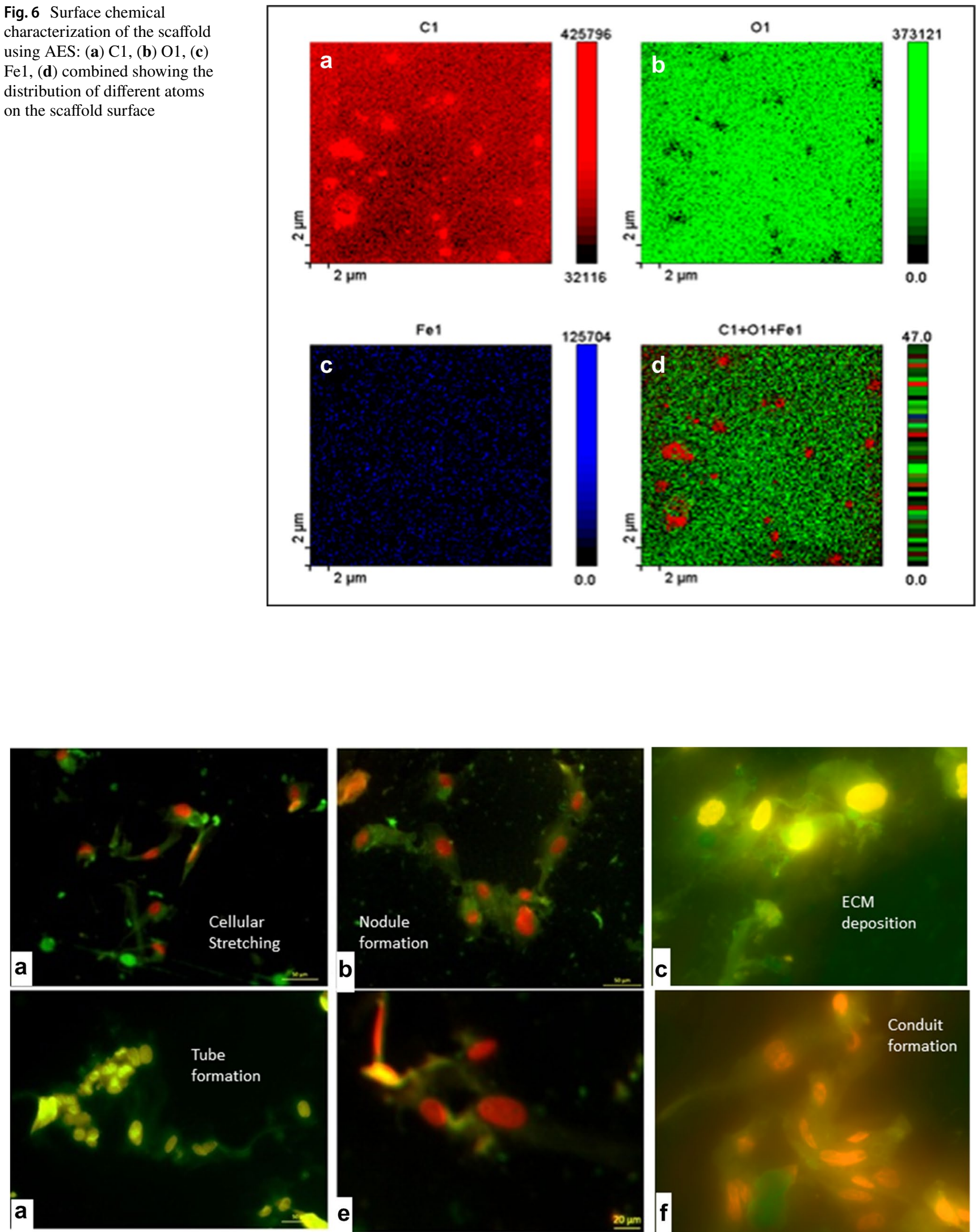

Fig. 7 Live cell microscopic imaging (7 days). (a) Cellular stretching. (b) Bone-like nodule formation. (c) Thick ECM deposition. (d) Endothelial cell-like tube formation. (d and e) Conduit formation (a, b, d scale, $50 \mu \mathrm{m} ; \mathbf{c}, \mathbf{e}, \mathbf{f}$ scale, $20 \mu \mathrm{m}$ ) 
Fig. 8 PCR analysis of gene expression after 15 days of cell culture showing high osteocalcin and PECAM expression suggesting both osteogenic and endothelial gene differentiation pathway activation of the MSCs $(n=3, p \leq 0.05)$

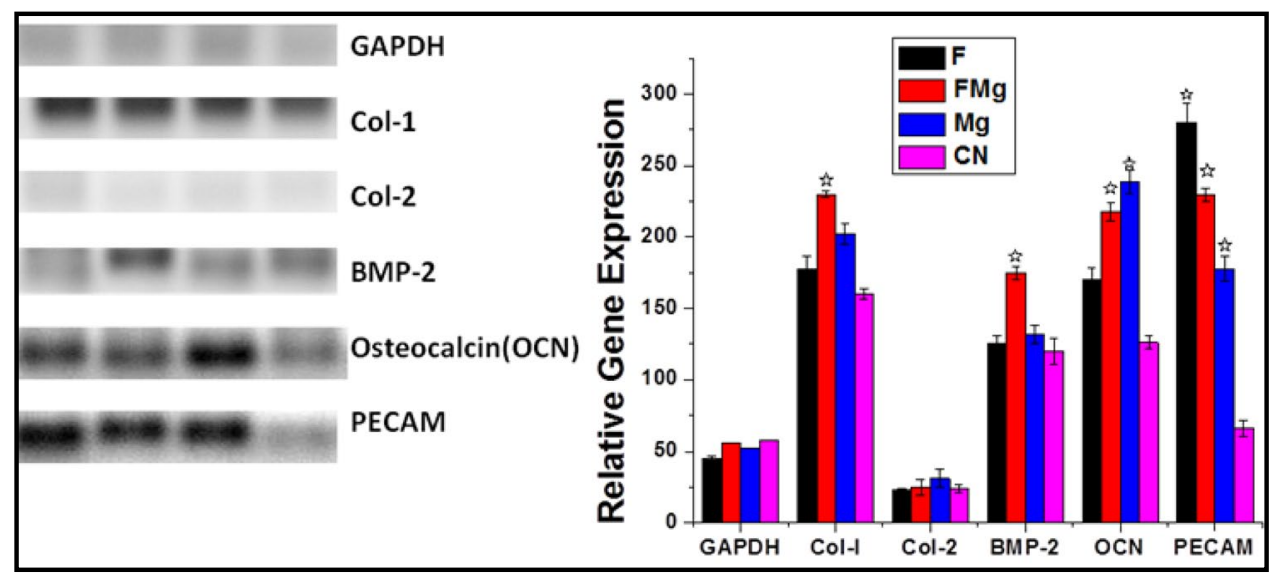

double-sided adhesive tape. Hence, the mechanical stretch in the scaffold generated micro-tension to the adherent MSCs in the scaffolds. This micro-tension is significantly effective in osteogenic differentiation of MSCs (bone-like phenotype development). Literature suggests the mechanical stress in the order of $0.3-0.8 \mathrm{MPa}$ is significantly effective for bone-like differentiation. [38] The current model has mechanical stiffness in the range of ECM of trabecular bone. But, at steady state, the shear gradient on the cells is in the range of endothelial differentiation. With almost control equivalent collagen- 2 expression, it is also evident that the bone development phenomenon is similar to intramembranous ossification. It is already evident in previous biomechanical studies that combination of tensile strain and fluid shear gradient can lead to intramembranous osteogenesis. [39, 40] Intramembranous ossification is the natural fracture healing mechanism in the human body. [41] To create a similar system in vitro requires activation of an inflammatory microenvironment via cytokines like IL-1 $\beta$, TNF- $\alpha$, and IFN-Y (NF-K $\beta$ activation). [42] The only other way to develop such models is via development of a coculture of bone cells and pro-inflammatory (M1) macrophages.[43] Thus, modeling this system is significantly important and economically viable especially for testing therapeutics related to flat bone healing like the skull or mandible [44].

\section{Conclusion}

The current study shows that exploring biophysical stimuli can be an alternative to growth factors for modeling complex organ systems. In the developed model, we not only mimic vascularization and osteogenesis in the same experimental chip via competition of two orthogonal mechanical forces, but also find mechanistic similarities between nature's natural fracture healing mechanism (intramembranous ossification). In this model, no growth factors are utilized and the physical property can be controlled easily (modulation of nanoparticle content, changing magnet strength, changing the channel dimensions, and changing the fluid input velocity). Thus, this experimental model could be significantly useful in exploring therapeutics involved in fracture healing. However, a major limitation of this system is creating its mathematical model so that it can be used to deal with complex bioengineering problems. Here, two different forces are interacting and one of them cannot be calculated directly (magnetic actuation). So, multiplexing the system and creating better mathematical model could enhance the scope of the device.

Supplementary Information The online version contains supplementary material available at https://doi.org/10.1007/s44164-021-00004-7.

Data availability The data is available in electronic supplementary document.

\section{Declarations}

Conflict of interest The authors declare no competing interests.

\section{References}

1. Feng X, McDonald JM. Disorders of bone remodeling. Annu Rev Pathol. 2011;6:121-45. https://doi.org/10.1146/annur ev-pathol-011110-130203.

2. Dadhich P, et al. A simple approach for an eggshell-based 3D-printed osteoinductive multiphasic calcium phosphate scaffold. ACS Appl Mater Interfaces. 2016;8:11910-24. https://doi. org/10.1021/acsami.5b11981.

3. Bhatia SN, Ingber DE. Microfluidic organs-on-chips. Nat Biotechnol. 2014;32:760-72. https://doi.org/10.1038/nbt.2989.

4. Arrigoni C, Gilardi M, Bersini S, Candrian C, Moretti M. Bioprinting and organ-on-chip applications towards personalized medicine for bone diseases. Stem cell reviews and reports. 2017;13:407-17. https://doi.org/10.1007/s12015-017-9741-5.

5. Bongio M, Lopa S, Gilardi M, Bersini S, Moretti M. A 3D vascularized bone remodeling model combining osteoblasts and 
osteoclasts in a $\mathrm{CaP}$ nanoparticle-enriched matrix. Nanomedicine. 2016;11:1073-91. https://doi.org/10.2217/nnm-2015-0021.

6. Truesdell SL, George EL, Saunders MM. Cellular considerations for optimizing bone cell culture and remodeling in a labon-a-chip platform. Biotechniques. 2020. https://doi.org/10.2144/ btn-2019-0115.

7. Bersini $\mathrm{S}$, et al. Human in vitro $3 \mathrm{D}$ co-culture model to engineer vascularized bone-mimicking tissues combining computational tools and statistical experimental approach. Biomaterials. 2016;76:157-72. https://doi.org/10.1016/j.biomaterials.2015.10. 057.

8. Heo DN, Hospodiuk M, Ozbolat IT. Synergistic interplay between human MSCs and HUVECs in 3D spheroids laden in collagen/ fibrin hydrogels for bone tissue engineering. Acta Biomater. 2019;95:348-56. https://doi.org/10.1016/j.actbio.2019.02.046.

9. Chramiec A, Vunjak-Novakovic G. Tissue engineered models of healthy and malignant human bone marrow. Adv Drug Deliv Rev. 2019;140:78-92. https://doi.org/10.1016/j.addr.2019.04.003.

10. Mei X, et al. Microfluidic platform for studying osteocyte mechanoregulation of breast cancer bone metastasis. Integrative biology : quantitative biosciences from nano to macro. 2019;11:11929. https://doi.org/10.1093/intbio/zyz008.

11 Masters EA, et al. An in vitro platform for elucidating the molecular genetics of S. aureus invasion of the osteocyte lacuno-canalicular network during chronic osteomyelitis. Nanomedicine. 2019;21:102039. https://doi.org/10.1016/j.nano.2019.102039.

12. Aleman J, et al. Deconstructed microfluidic bone marrow on-achip to study normal and malignant hemopoietic cell-niche interactions. 2019;15:e1902971. https://doi.org/10.1002/smll.20190 2971.

13. Almeida-Porada, G. Small, https://doi.org/10.1002/smll.20190 2971.

14. Sakolish C, et al. Tissue-engineered bone tumor as a reproducible human in vitro model for studies of anticancer drugs. Toxicol Sci. 2020;173:65-76. https://doi.org/10.1093/toxsci/kfz220.

15. Fuller HC, Wei T-Y, Behrens MR, Ruder WC. The future application of organ-on-a-chip technologies as proving grounds for MicroBioRobots. 2020;11:947.

16. Zhang YS, et al. Multisensor-integrated organs-on-chips platform for automated and continual in situ monitoring of organoid behaviors. Proc Natl Acad Sci. 2017;114:E2293. https://doi.org/ 10.1073/pnas.1612906114.

17. Harshad K, et al. An electromagnetic cell-stretching device for mechanotransduction studies of olfactory ensheathing cells. Biomed Microdevice. 2016;18:45. https://doi.org/10.1007/ s10544-016-0071-1.

18. Mi S, Pu H, Xia S, Sun W. A minimized valveless electromagnetic micropump for microfluidic actuation on organ chips. Sensors and Actuators A: Physical. 2020;301:111704. https://doi.org/10. 1016/j.sna.2019.111704.

19. George EL, Truesdell SL, Magyar AL, Saunders MM. The effects of mechanically loaded osteocytes and inflammation on bone remodeling in a bisphosphonate-induced environment. Bone. 2019;127:460-73. https://doi.org/10.1016/j.bone.2019.07.008.

20. George EL, Truesdell SL, York SL, Saunders MM. Lab-on-a-chip platforms for quantification of multicellular interactions in bone remodeling. Exp Cell Res. 2018;365:106-18. https://doi.org/10. 1016/j.yexcr.2018.02.027.

21. Nature biomedical engineering, https://doi.org/10.1038/ s41551-019-0495-z.

22. Lin Z, et al. Osteochondral tissue chip derived from iPSCs: modeling OA pathologies and testing drugs. Frontiers in bioengineering and biotechnology. 2019;7:411. https://doi.org/10.3389/fbioe. 2019.00411.

23. Oftadeh, R., Perez-Viloria, M., Villa-Camacho, J. C., Vaziri, A. \& Nazarian, A. Biomechanics and mechanobiology of trabecular bone: a review. J Biomech Eng. 2015:137. https:// doi.org/10.1115/1.4029176.

24. Singer FR. Bone quality in Paget's disease of bone. Curr Osteoporos Rep. 2016;14:39-42. https://doi.org/10.1007/ s11914-016-0303-6.

25. Dong J-D, et al. Response of mesenchymal stem cells to shear stress in tissue-engineered vascular grafts. Acta Pharmacol Sin. 2009;30:530-6. https://doi.org/10.1038/aps.2009.40.

26. Qi MC, et al. Mechanical strain induces osteogenic differentiation: Cbfa1 and Ets-1 expression in stretched rat mesenchymal stem cells. Int J Oral Maxillofac Surg. 2008;37:453-8. https:// doi.org/10.1016/j.ijom.2007.12.008.

27. Li R, et al. Mechanical strain regulates osteogenic and adipogenic differentiation of bone marrow mesenchymal stem cells. Biomed Res Int. 2015;2015:873251-873251. https://doi.org/10. $1155 / 2015 / 873251$.

28. du Toit H, Macdonald TJ, Huang H, Parkin IP, Gavriilidis A. Continuous flow synthesis of citrate capped gold nanoparticles using UV induced nucleation. RSC Adv. 2017;7:9632-8. https:// doi.org/10.1039/C6RA27173A.

29. Das B, Pal P, Dadhich P, Dutta J, Dhara S. In vivo cell tracking, reactive oxygen species scavenging, and antioxidative gene down regulation by long-term exposure of biomass-derived carbon dots. ACS Biomater Sci Eng. 2019;5:346-56. https://doi. org/10.1021/acsbiomaterials.8b01101.

30. Das B, Dadhich P, Pal P, Dhara S. Single step synthesized sulfur and nitrogen doped carbon nanodots from whey protein: nanoprobes for longterm cell tracking crossing the barrier of photo-toxicity. RSC Adv. 2016;6:60794-805. https://doi.org/10. 1039/C5RA25506F.

31. Krispin M, Ullrich A, Horn S. Crystal structure of iron-oxide nanoparticles synthesized from ferritin. J Nanopart Res. 2012;14:669. https://doi.org/10.1007/s11051-011-0669-4.

32. Das B, et al. Carbon nanodots doped super-paramagnetic iron oxide nanoparticles for multimodal bioimaging and osteochondral tissue regeneration via external magnetic actuation. ACS Biomater Sci Eng. 2019;5:3549-60. https://doi.org/10.1021/ acsbiomaterials.9b00571.

33. Majhy B, Priyadarshini P, Sen AK. Effect of surface energy and roughness on cell adhesion and growth - facile surface modification for enhanced cell culture. RSC Adv. 2021;11:15467-76. https://doi.org/10.1039/D1RA02402G.

34 Zysset PK, Edward Guo X, Edward Hoffler C, Moore KE, Goldstein SA. Elastic modulus and hardness of cortical and trabecular bone lamellae measured by nanoindentation in the human femur. Journal of Biomechanics. 1999;32:1005-12. https://doi. org/10.1016/S0021-9290(99)00111-6.

35 Mansoorifar A, Gordon R, Bergan RC, Bertassoni LE. Bone-on-a-chip: microfluidic technologies and microphysiologic models of bone tissue. Advanced Functional Materials. 2021;31:2006796. https://doi.org/10.1002/adfm.202006796.

36. Griffith $\mathrm{CK}$, et al. Diffusion limits of an in vitro thick prevascularized tissue. Tissue Eng. 2005;11:257-66. https://doi.org/10. 1089/ten.2005.11.257.

37. Kim HW, Lim J, Rhie JW, Kim DS. Investigation of effective shear stress on endothelial differentiation of human adiposederived stem cells with microfluidic screening device. Microelectron Eng. 2017;174:24-7. https://doi.org/10.1016/j.mee. 2016.12.022.

38. McCoy RJ, O'Brien FJ. Influence of shear stress in perfusion bioreactor cultures for the development of three-dimensional bone tissue constructs: a review. Tissue engineering Part B, Reviews. 2010;16:587-601. https://doi.org/10.1089/ten.TEB. 2010.0370.

39. Claes LE, Heigele CA. Magnitudes of local stress and strain along bony surfaces predict the course and type of fracture healing. J 
Biomech. 1999;32:255-66. https://doi.org/10.1016/S00219290(98)00153-5.

40. Lacroix D, Prendergast PJ. A mechano-regulation model for tissue differentiation during fracture healing: analysis of gap size and loading. J Biomech. 2002;35:1163-71. https://doi.org/10.1016/ S0021-9290(02)00086-6.

41. Huang C, Ogawa R. Mechanotransduction in bone repair and regeneration. FASEB J. 2010;24:3625-32. https://doi.org/10. 1096/fj.10-157370.

42. Kon T, et al. Expression of osteoprotegerin, receptor activator of NF-kappaB ligand (osteoprotegerin ligand) and related proinflammatory cytokines during fracture healing. Journal of bone and mineral research : the official journal of the American Society for
Bone and Mineral Research. 2001;16:1004-14. https://doi.org/10. 1359/jbmr.2001.16.6.1004.

43. Tu M-G, Chen Y-W, Shie M-Y. Macrophage-mediated osteogenesis activation in co-culture with osteoblast on calcium silicate cement. J Mater Sci - Mater Med. 2015;26:276. https://doi.org/ 10.1007/s10856-015-5607-z.

44 Tong L, Buchman SR, Ignelzi MA Jr, Rhee S, Goldstein SA. Focal adhesion kinase expression during mandibular distraction osteogenesis: evidence for mechanotransduction. Plast Reconstr Surg. 2003;111:211-22. https://doi.org/10.1097/01.prs.0000033180. 01581.9a (discussion 223-214). 